\title{
Effectiveness of ${ }^{125}$ I seed implantation in the treatment of non-small cell lung cancer during $R 2$ resection
}

\author{
WEI LI ${ }^{1}$, YIFENG ZHENG ${ }^{1}$, YUNMING LI $^{2}$, JING GUAN ${ }^{3}$, \\ JIANQING JIANG $^{1}$, YONGKANG YU ${ }^{1}$, XIUSHAN ZHENG ${ }^{1}$ and LIE YANG $^{1}$ \\ ${ }^{1}$ Department of Thoracic Surgery; ${ }^{2}$ Information Centre; ${ }^{3}$ Department of Radiology, \\ General Hospital of Chengdu Military Region, Chengdu 610083, P.R. China
}

Received February 26, 2016; Accepted July 20, 2017

DOI: 10.3892/ol.2017.7019

\begin{abstract}
The aim of the present study was to investigate the effectiveness of ${ }^{125} \mathrm{I}$ particle implantation during R2resection for non-small cell lung cancer (NSCLC). Data from 23 patients with NSCLC and macroscopic residual diseasefollowing surgery (R2 resection) between March 2010 and May 2014 were retrospectively analyzed. Among these patients, 12 patients [4 with T-residual disease (incomplete resection of primary tumor but complete dissection of regional lymph node), 8 with N-residual disease (complete resection of primary tumor but incomplete resection of metastatic regional lymph node)] underwent ${ }^{125}$ I particle implantation during the operation, while the other 11 (4 with T-residual disease and 7 with $\mathrm{N}$-residual disease) received postoperative conventional radiotherapy. The local control rate, overall survival, and distant metastasis were evaluated. Additionally, the efficacy and safety of brachytherapy using ${ }^{125} \mathrm{I}$ particle implantation during surgery for locally advanced NSCLC were investigated. The 23 patients were followed up for 3-40 months. For the ${ }^{125}$ I group, the 2-year local control rate was $100 \%$, and the median survival time was 24 months. The 1-2-year survival rates were 83.3 and $58.33 \%$, respectively. For the postoperative radiotherapy group, the median survival time was 12 months, andthe 1- and 2-year survival rates were 54.5 and $27.7 \%$, respectively. No statistically significant difference in 2-year survival rates was detected between the two treatment groups, but the particle implantation group exhibited a higher survival rate trend. For patients with T-residual disease, the survival rate was higher for the ${ }^{125} \mathrm{I}$ seed implantation group compared with the postoperative radiotherapy group. However, there was no significant difference in the rates of metastasis between the two groups for patients with N-residual disease. Therefore,
\end{abstract}

Correspondence to: Dr Yifeng Zheng, Department of Thoracic Surgery, General Hospital of Chengdu Military Region, 270 City Rong Road, Chengdu 610083, P.R. China

E-mail: yongge5@163.com

Key words: ${ }^{125}$ I seed, brachytherapy, intraoperative implantation, lung cancer, $\mathrm{R} 2$ resection intraoperative implantation of ${ }^{125} \mathrm{I}$ particles during $\mathrm{R} 2$ resection of NSCLC may be a safer and more reliable method to reduce the local recurrence rate compared with conventional radiotherapy. Although not statistically significant, the overall survival rate of patients in the ${ }^{125}$ I seed implantation group was higher compared with the postoperative radiotherapy group.

\section{Introduction}

Lung cancer is one of the most common types of cancer worldwide and hasone of the highest mortality rates (1). Despite advances in imaging technologies, which have substantially improved the accuracy of preoperative staging of lung cancer, the extent of the disease remains underestimated, and radical surgical resection may not be feasible following exploratory surgery. The present study aimed at investigating intraoperative patients with residual cancer. Implantation of ${ }^{125}$ I may control residual disease, and reduce the risk of surgery and postoperative complications. Otherwise, post-surgical radiotherapy is required to control the residual site of tumor growth (2). Previous studies have indicated that the rate of exploratory surgery decreases gradually with time to between 1 and $2 \%$, or even lower $(2,3)$. However, the incidence of macroscopic residual disease (R2) following resection of non-small cell lung cancer (NSCLC) remains at $\sim 4 \%$ (3). Intra-tumor ${ }^{125} \mathrm{I}$ implantation is a localized radiotherapy and any adverse effects on normal tissues are confined to the immediate vicinity (1). Furthermore, the intra-tumor ${ }^{125}$ I may reach the prescribed radioactivity between 110 and 160 Gy locally, which is considered to be a curable radiation dose and lasts for a longer period of time, compared with conventional external beam radiation to eliminate the tumor cells (4). External irradiation is generally administered following surgery, although the efficacy of this process is suboptimal, without any marked improvement in survival (3). Intraoperative implantation of irradiative particles increases the local control in patients with locally advanced lung cancer.

In the present study, 12 patients with macroscopic residual disease following exploratory surgery received radical surgical resection plus intraoperative implantation of ${ }^{125} \mathrm{I}$ seeds between March 2010 and May 2014. The duration of treatment, local recurrence, median survival time and median progression free survival (PFS) were evaluated. 


\section{Materials and methods}

Clinical data. Between March 2010 and May 2014, 23 patients with NSCLC (17 males and 6 females) were included, from General Hospital of Chengdu Military Region, in the present study. 12 patients in the radioactive seed implant group and 11 patients the conventional radiotherapy group. The clinical data of the patients are shown in Table I. The indications for ${ }^{125}$ I particle implantation were as follows: i) Stage T4 disease, according to the 7th Japan Joint Committee of Lung Cancer/Union for International Cancer Control Tumor Node Metastasis staging system for NSCLC (5). was identified during treatment and radically resected; ii) the location of the lesion was at the pulmonary hilus. The residual lesion infiltrated the major blood vessels, which prevents safe resection; iii) the lesions involved the mediastinum, trachea, esophagus, aorta, superior vena cava or pericardium; and iv) tumor invasion of the thoracic walls or spine preventing complete removal. The following exclusion criteria was applied: i) Mortality within 30 days' post-surgery; ii) aged $>80$ years; and iii) lung tumors were non-primary lesions. The treatment methods were agreed upon by the patients, and informed consent was provided from all patients. The present study was approved by the Ethics Committee of the General Hospital of Chengdu Military Region (approval no. 10-00253) (Chengdu, China), and written informed consent was obtained from each participant.

Materials. ${ }^{125}$ I radioactive seeds with $22.4-29.6 \mathrm{MBq} /$ particle were obtained from Shanghai GMS Pharmaceutical Co., Ltd. (Shanghai, China). An enclosed rotatory implanter and implant needles [Hakko International Trading (Shanghai) Co., Ltd., Shanghai, China] were used to implant the radioactive seeds.

Methods. Tumors of the 23 patients scheduled for thoracotomy were resected. The lymph nodes with residual disease were validated by frozen section examination (Fresh tissue frozen for 5 min under -20 degrees Celsius, $4 \%$ formaldehyde fixation for $10-15 \mathrm{sec}$, hematoxylin staining for $30 \mathrm{sec}$, eosin staining for $3 \mathrm{sec}$ after hydrochloric alcohol differentiation for $1 \mathrm{sec}$, rinse water, neutral resin sheet. Observe under OLYMPUS microscope (X10). From tissue sections removed during surgery. Once the sites of residual disease were identified, the particles were implanted according to the Radiotherapy Treatment Planning System (TPS) plan with a dose of 120 Gy (1). Postoperative external irradiation (40-60 Gy at a dose of 2 Gy/day for 20-30 days) was used in patients in the conventional treatment group within two months of treatment.

Intraoperative particle implantations. Images of the areas of residual disease were captured and the thickness of the residual lymph nodes was measured. This data were subsequently inputted into the TPS system for 3D reconstruction. The overall dosage and the number of particles required for tumor control were calculated. The volume of the residual tumor was estimated and recorded in the TPS system to simulate the size of the lymph node (Fig. 1). A particle was implanted every $0.5-1.0 \mathrm{~cm}$, and the target dose was $120 \mathrm{~Gy}$. The volume of the residual lesions were estimated, and the 22.4-29.6MBq/particles were implanted $0.5-1.0 \mathrm{~cm}$ to maintain identical distances between the particles. For tumor tissues with relatively low thickness, a gelatin sponge was used as the particles were not implanted deeply enough. The particles were distributed at a pitch of $1 \mathrm{~cm}$ in the form of gelatin sponge, and were attached to the residual surface (6) For the cancerous residual disease tissues on the bronchial stump, a fine thread was used to directly suture the particles onto the tumor tissues. In the 12 patients, a total of 4 to $30{ }^{125}$ I particles were implanted, with an average of 10 per patient. A total of 6 patients received 2-6 cycles of postoperative chemotherapy. Appropriate TPS plans were developed according to the shape and size of residual lesions, so that the particle implant dose distribution was more uniform between the particles.

Follow-up. All 23 patients entered the follow-up phase immediately following resection. The intended follow-up period was 40 months with visits at 1 month, 3 months and every 3 months thereafter. Anteroposterior and lateral chest images and computed tomography (CT) scans were conducted one week following the surgery to assess the distribution of particles. The patients were re-examined at 1, 2,6 and 12 months and every 6 months thereafter. Following surgery, clinical examination, blood sampling and $\mathrm{CT}$ examination of the chestwere performed. No patients were lost to follow-up. Follow-up chest CT scans were obtained to evaluate response following surgery.

Statistical analysis. SPSS software (version 16.0; SPSS, Inc., Chicago, IL, USA) was used to perform statistical analysis. Qualitative data are described as frequency and percentage, and qualitative data are expressed as the mean \pm standard deviation. $\chi^{2}$ test was used to compare the qualitative data, while an unpaired t-test was used for the comparison of quantitative data between the implantation and conventional groups. The Kaplan-Meier method was used to perform survival analysis, and log-rank test was used to compare the survival time between the two groups. $\mathrm{P}<0.05$ was considered to indicate a statistically significant difference. The survival rate, PFS, median survival time, and the percentage of T-/N-residual disease were compared between the two groups.

\section{Results}

Patient survival. The characteristics of the patients are listed in Table I. The median follow-up time was 18 months for all patients and 24 months for the patients in the ${ }^{125} \mathrm{I}$ particle implant group (Table II). In total, the patients were followed up for 1 to 4 and, 2 years after surgery, no tumor recurrence was observed in the patients who underwent ${ }^{125} \mathrm{I}$ particle implantation. The 2-year local control rate was $100 \%$. The local control rate in the ${ }^{125}$ I particle implantation group was markedly higher compared with patients who underwent postoperative external irradiation, irrespective of $\mathrm{T}$ - or N2-residual disease. The median survival time was 12 months and the local recurrence rate was $54.4 \%$.

In the ${ }^{125}$ I particle implantation group, 4 patients succumbed to pulmonary failure, and 3 succumbed to brain metastases. In addition, 2 patients were found with thoracic, abdominal and bone metastases. Of the 3 surviving patients, multiple lymph node metastases around the lesion were observed in 1 patient, with postoperative pathological examination indicating $\mathrm{N} 2$-stage disease. The lymph nodes at the ascending aorta and 
Table I. Patient characteristics.

\begin{tabular}{|c|c|c|c|}
\hline Characteristics & Radioactive seed implant group $(\mathrm{n}=12)$ & Conventional radiotherapy group $(n=11)$ & P-value \\
\hline Age & & & 0.263 \\
\hline Range, years & $44-69$ & $37-73$ & \\
\hline Mean $\pm \mathrm{SD}$, years & $57.92 \pm 7.57$ & $53.00 \pm 12.55$ & \\
\hline Sex & & & 0.901 \\
\hline Male & 9 & 8 & \\
\hline Female & 3 & 3 & \\
\hline Histology & & & 0.624 \\
\hline Squamous & 4 & 6 & \\
\hline Adenocarcinoma & 6 & 4 & \\
\hline Adenosquamous & 2 & 1 & \\
\hline Adenosquamous & 0 & 0 & \\
\hline \multicolumn{4}{|l|}{ Carcinoma } \\
\hline TNM classification ${ }^{\mathrm{a}}$ & & & 0.572 \\
\hline IIA & 2 & 3 & \\
\hline IIB & 1 & 2 & \\
\hline IIIA & 9 & 6 & \\
\hline Classification of tumor & & & 0.879 \\
\hline T-R2 type & 4 & 4 & \\
\hline N-R2 type & 8 & 7 & \\
\hline Size of tumor ${ }^{\mathrm{b}}, \mathrm{cm}^{3}$ & $66.42 \pm 70.41$ & $100.45 \pm 208.03$ & 0.598 \\
\hline Chemotherapy regimen & & & 0.481 \\
\hline GEM+DDP & 3 & 5 & \\
\hline $\mathrm{PC}$ & 5 & 3 & \\
\hline Other & 4 & 3 & \\
\hline
\end{tabular}

${ }^{a}$ Union for International Cancer Control tumor-node-metastasis staging system; ${ }^{\mathrm{b}}$ Mean \pm SD. SD, standard deviation; GEM, gemcitabine; DDP, cisplatin; PC, pemetrexed andcarboplatin; N-R2 type, complete resection of primary tumor but incomplete resection of metastatic regional lymph node with macroscopic residual tumor; T-R2 type, incomplete resection, with macroscopic residual primary tumor, but complete dissection of regional lymph node.
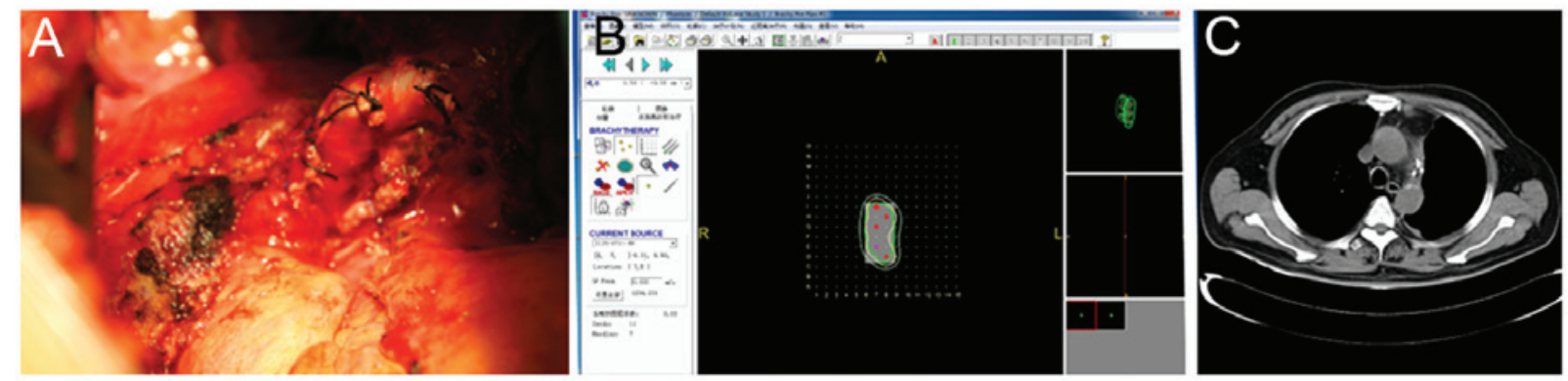

Figure 1. Example of a patient with left upper adenocarcinoma. During the operation, residual lymph nodes under the aortic arch were detected. The lymph nodes were fixed, and the lesion was observed to invade the pericardium. Partial pericardiotomy was performed, and intraoperative ${ }^{125}$ I particles implantation was performed via TPS system to obtain a dosage of $120 \mathrm{~Gy}$ in the target region. The regions in the pericardium with suspicious cancer residuals were implanted with ${ }^{125}$ I particles. (A) Image showing theinvasion of the pericardium by a metastatic lymph node. (B) Intraoperative ${ }^{125}$ I particles implantation was performed via the treatment plan system to obtain a dosage of $120 \mathrm{~Gy}$ at the target region. (C) The distribution of the particles was confirmed to be satisfactory computed tomography at 1 month post-operation.

aortic window were fixed, and a total of $9{ }^{125} \mathrm{I}$ particles were implanted during the operation. Another patient was found to have brain metastases 3 months following the operation, and was treated with stereotactic radiotherapy.
In the conventional treatment group, the 11 patients that underwent conventional treatment for only one cycle, 40-60 Gy of external irradiation (2 Gy/day for 20-30 days) was used postoperatively for the target region. The median survival 


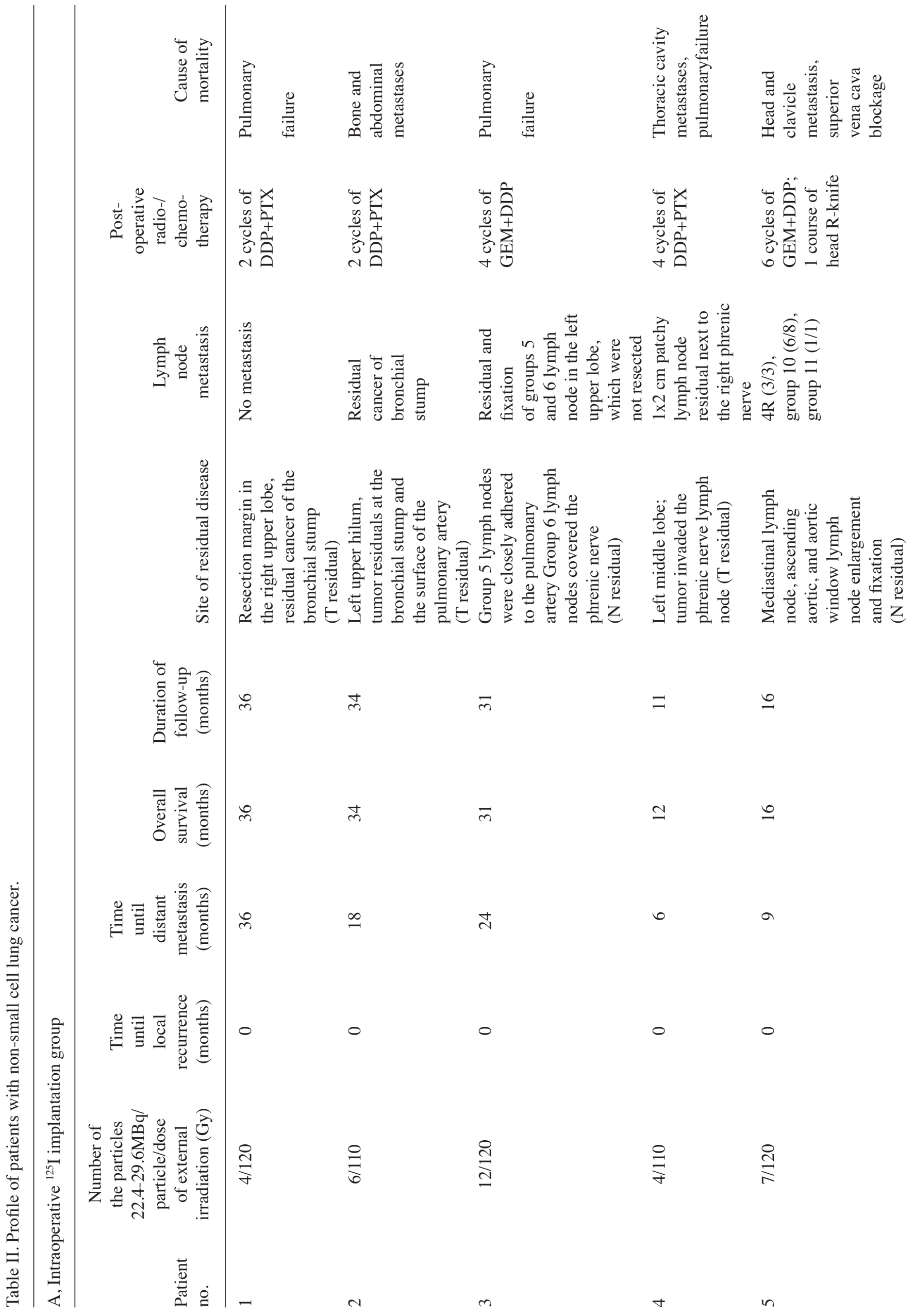




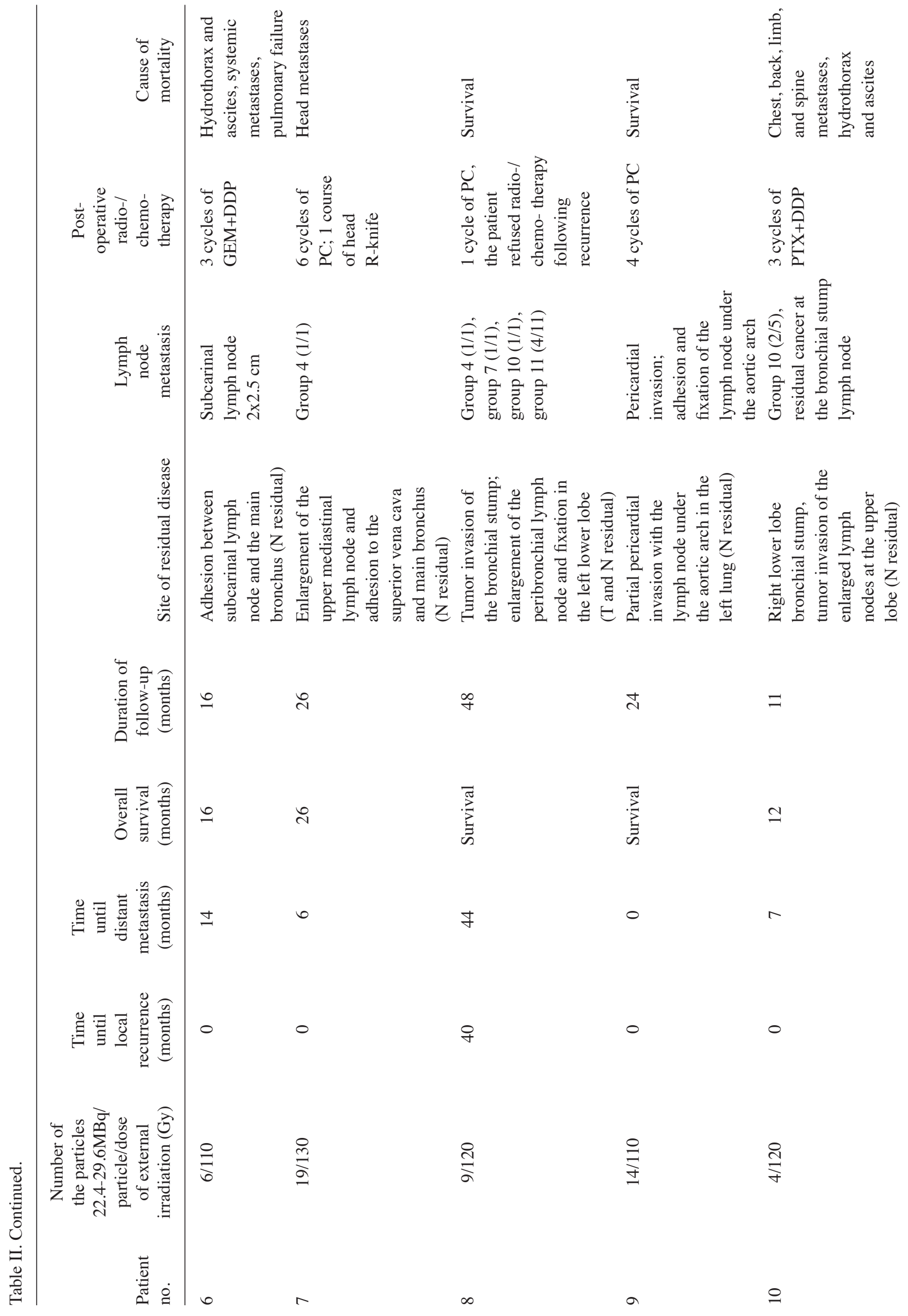




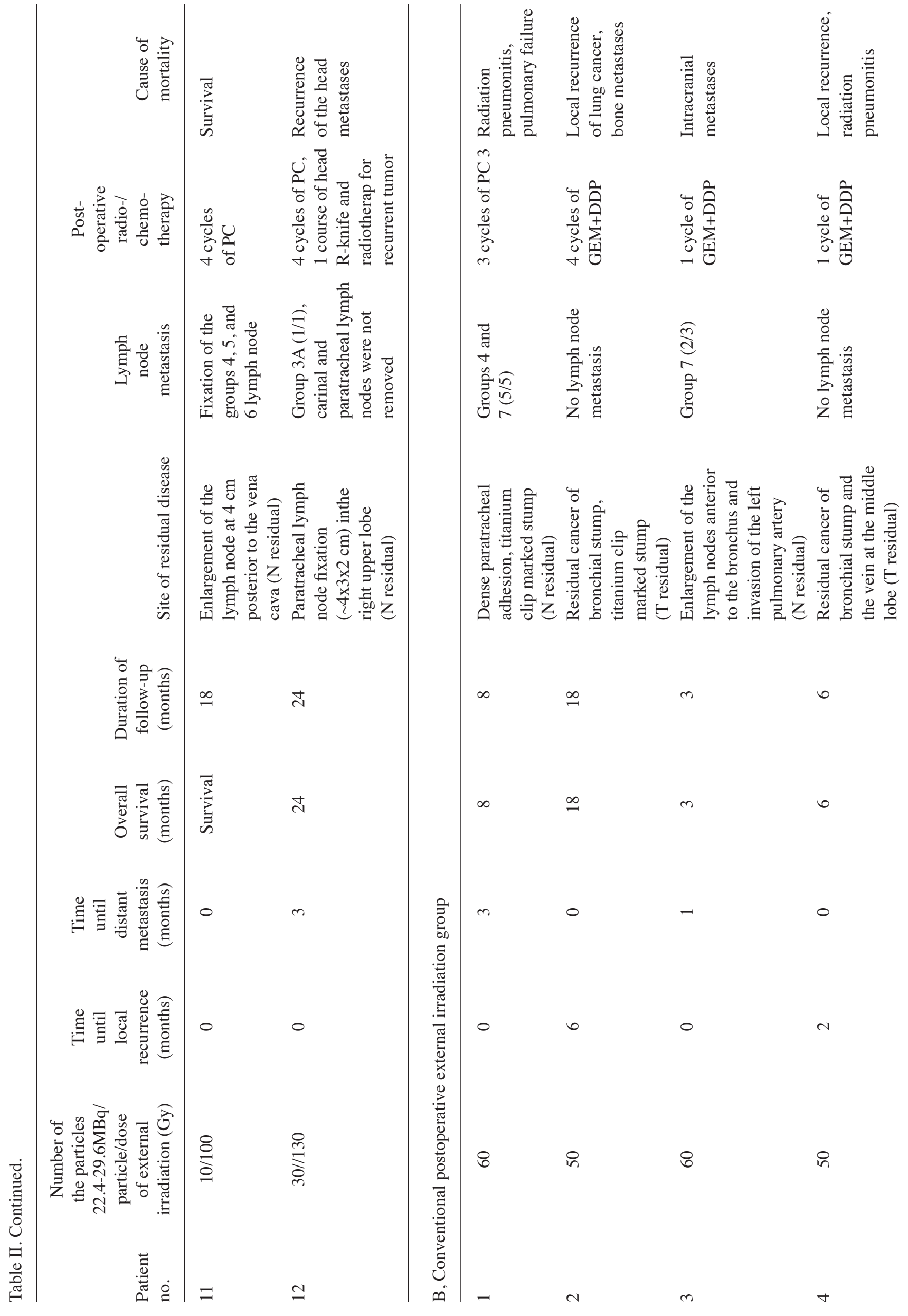




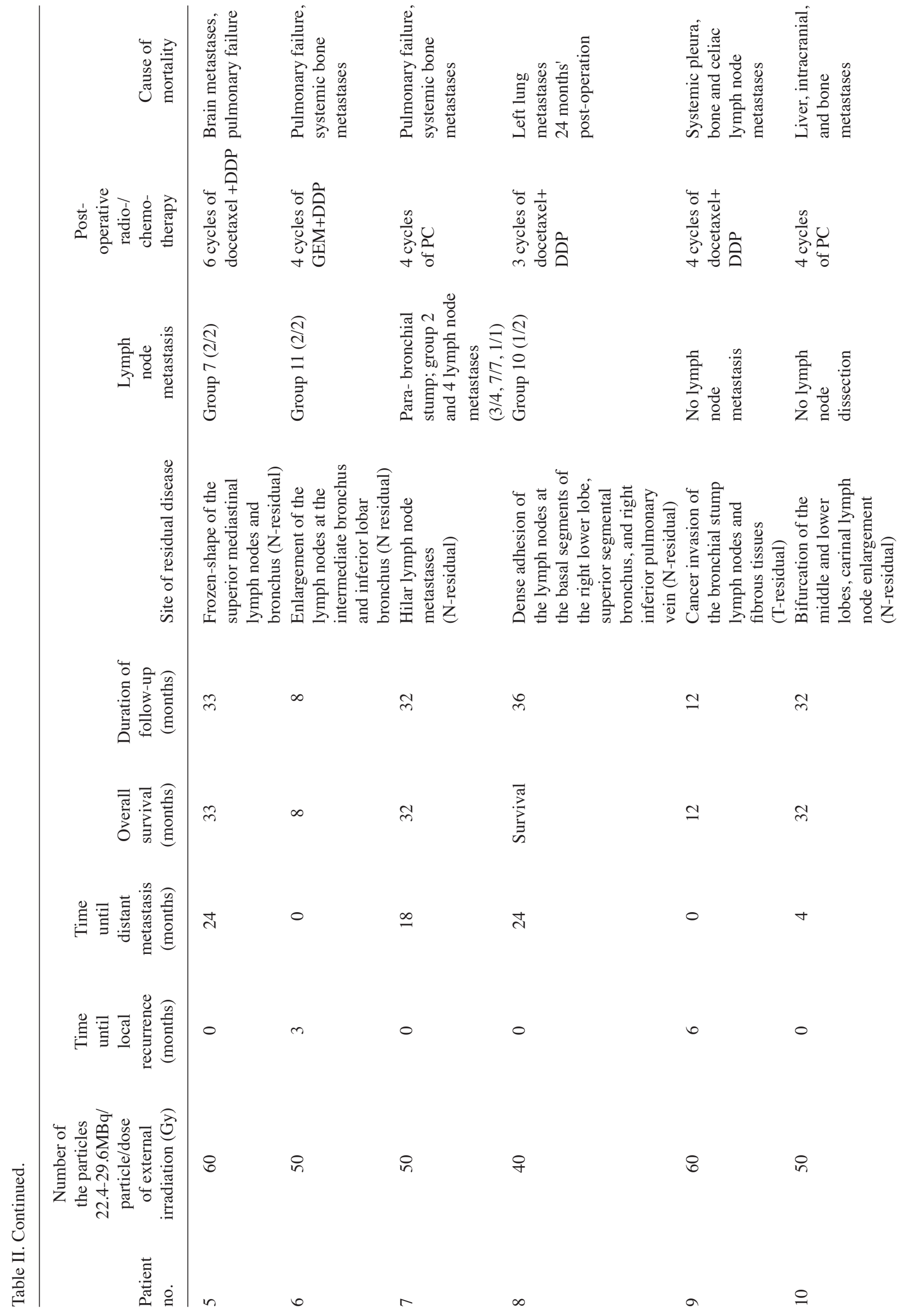


time of these 11 patients was 12 months. Of the 11 patients, 1 survived, 4 succumbed to local recurrence, 2 of which were complicated by radiation pneumonitis and 6 succumbed to distant metastases, including brain, liver, renal and bone metastases.

Control rate of local and distant metastasis. All 23 patients underwent surgical resection, and the site of particle implantation was confirmed asR2 resection. A total of $121^{125}$ I particles were implanted in patients, including 4 patients with incomplete tumor resection with T-residual disease. In total, 8 patients exhibited lymph node metastases, with enlarged lymph nodes and invasion of the pulmonary artery, superior vena cava and the main bronchus. Of the patients undergoing conventional treatment, T-residual disease was observed in 4 patients and $\mathrm{N}$-residual disease was revealed in 7 patients. No tumor recurrence at the implantation site was observed 2 years following seed implantation in the 12 patients, who underwent ${ }^{125}$ I particle implantation. The local control rate was $100 \%$, and no patient succumbed to recurrence at the implantation site (Fig. 2).

Of the 11 patients who underwent conventional treatment, local recurrence was observed in 5 patients (Table II). The median survival time of the patients in the conventional radiotherapy group was 12 months, and the local recurrence rate was $45.4 \%$. The survival rates of patients in the conventional radiotherapy group and the radioactive seed implant group are indicated in Fig. 3. Details of the complications of the treatments for the patients in the two treatment groups are listed in Table III.

Details of an example case. Case 1 (Fig. 4) was a 72-year-old male patient. Macroscopic residual disease was observedin the left upper lobe. Intraoperative ${ }^{125} \mathrm{I}$ particle implantation was performed at the bronchial stump and the peri-pulmonary artery. The patient underwent intraoperative ${ }^{125}$ I particle implantation for squamous carcinoma of the left upper lobe, with residual diseasein the tissues adjacent to the left main bronchus. The left upper lobe was resected in October 2010, and a total of $12{ }^{125} \mathrm{I}$ particles $(29.6 \mathrm{MBq} /$ particle) were implanted at the site of the residual disease. No metastasis was identifiedat the implantation sites at follow-up 2 weeks following treatment, and the local control rate was satisfactory (Fig. 4).

\section{Discussion}

The incidence of macroscopic residual disease is $\sim 4 \%$ in NSCLC (3). Conventional radiochemotherapy is generally performed following incomplete resection of tumor tissue to prevent local recurrence and metastasis. However, for limited residual disease in vital organs, the benefit/risk ratio from external irradiation is relatively low.

In the present study, 23 patients were included, of whom 12 underwent ${ }^{125}$ I particle implantation. For the patients who underwent ${ }^{125} \mathrm{I}$ particle implantation, the 2-year local control rate was $100 \%$, and the median survival time was 24 months. Only one patient who underwent ${ }^{125} \mathrm{I}$ particle implantation exhibited recurrence near the implantation region at 40 months following surgery, and the other 8 patients were free from local recurrence after 2 years (the other 3 patients 
Table III. Comparison of the characteristics and outcomes of the radioactive seed implant group and the conventional radiotherapy group.

\begin{tabular}{lccc}
\hline Complications & Radioactive seed implant group $(\mathrm{n}=12)$ & Conventional radiotherapy group $(\mathrm{n}=11)$ & P-value \\
\hline Bronchopleural fistula & $0(0)$ & $0(0)$ & - \\
Great vessel rupture & $0(0)$ & $0(0)$ & - \\
Radiation pneumonitis & $0(0)$ & $3(27.3)$ & 0.093 \\
Dislocation of ${ }^{125}$ I particle & $1(8.3)$ & $0(0)$ & 0.522 \\
Bone marrow suppression & $1(8.3)$ & $3(27.3)$ & 0.317 \\
\hline
\end{tabular}

A

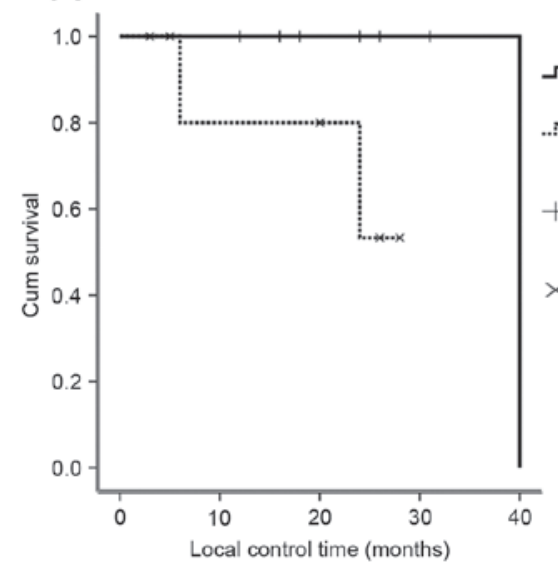

NR2

$\neg$ NR2 of iodine 125 implant

..." NR2 of

conventional radiotherapy

+ NR2 of iodine 125 implantcensored

$\times$ NR2 of

conventional radiotherapycensored

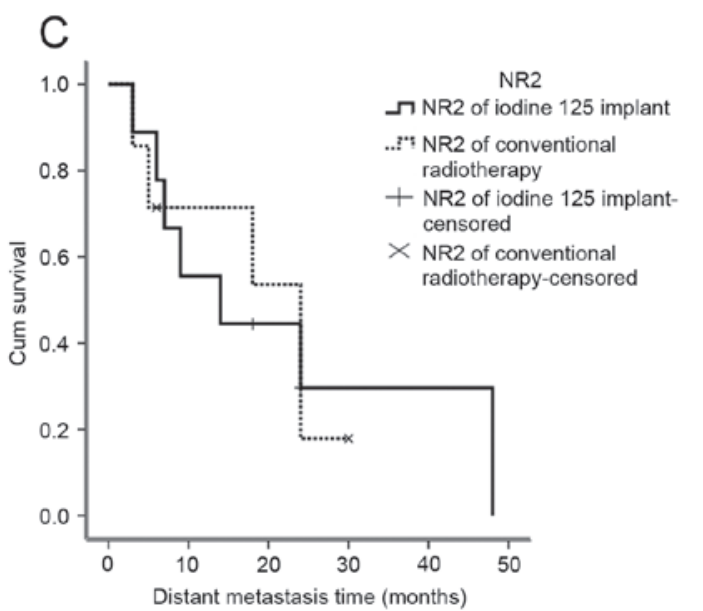

B
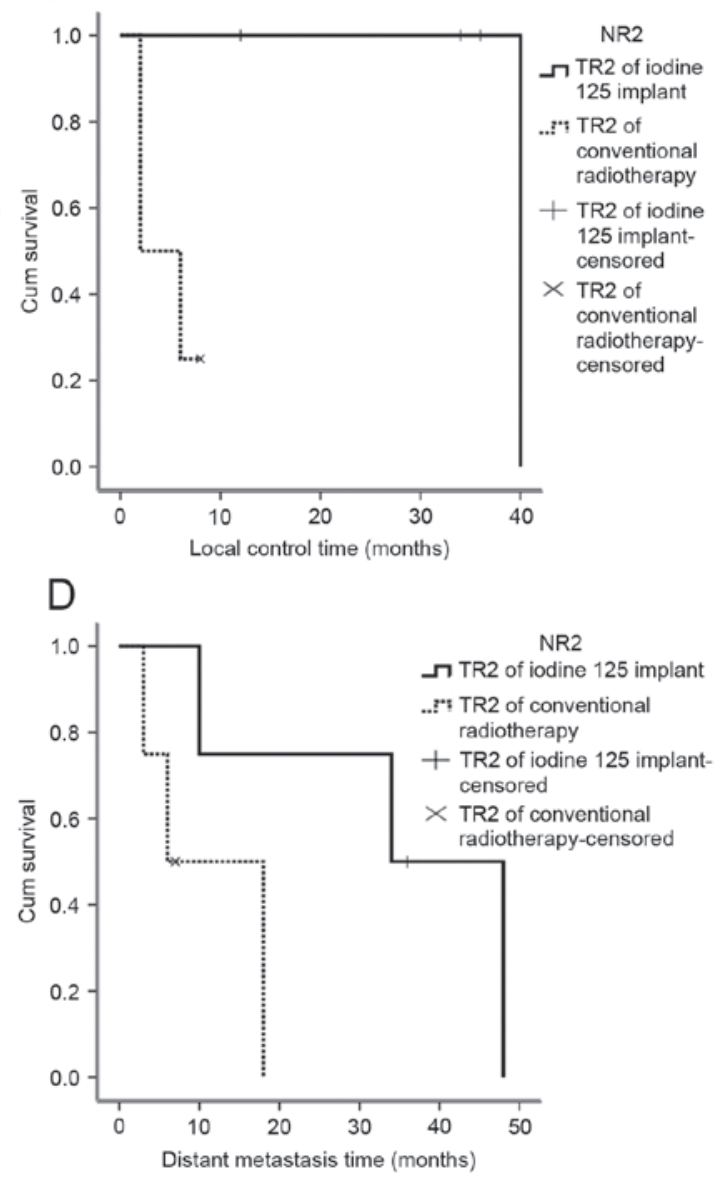

Figure 2. Comparison of the local control rate of patients with T-/N-residual disease in patients treated with ${ }^{125}$ I seed implantation or conventional radiotherapy. (A) The local control rate in the patients with N-residual disease. (B) The local control rate in the patients who underwent ${ }^{125}$ I particle implantation was significantly higher compared with conventional treatment $(\mathrm{P}<0.05)$. (C) Distant metastasis in patients with $\mathrm{N}$-residual disease was significantly lower in patients undergoing ${ }^{125} \mathrm{I}$ particle implantation compared with patients who underwent conventional treatment; (D) Distant metastasis in patients with T-residual disease. However, differences in patients with N-residual disease was not significantly different between the two groups. NR2, complete resection of primary tumor but incomplete resection of metastatic regional lymph node with macroscopic residual tumor; TR2, incomplete resection, with macroscopic residual primary tumor, but complete dissection of regional lymph node.

succumbed $<2$ years). The local control ratein the groups who underwent postoperative external irradiation group was $54.6 \%$. The local control rate in the ${ }^{125} \mathrm{I}$ particle implantation group was $100 \%$, and no patient succumbed to recurrence at the implantation site. The local control rate in the ${ }^{125} \mathrm{I}$ particle implantation group was significantly higher compared with patients who underwent postoperative external irradiation, irrespective of $\mathrm{T}$ - or $\mathrm{N} 2$ - residual disease $(\mathrm{P}<0.05)$. In addition, the 2-year survival rate was also higher in the
${ }^{125}$ I particle implantation group compared with the postoperative external irradiation group, although this difference was not statistically significant. Subgroup analysis revealed that in patients with simple T-residual disease, the survival rate in the ${ }^{125} \mathrm{I}$ particle implantation group was markedly higher compared with the postoperative external irradiation group, whereas the incidence of distant metastasis was markedly lower. However, for patients with N2-residual disease, the survival and metastasis rates were not significantly 


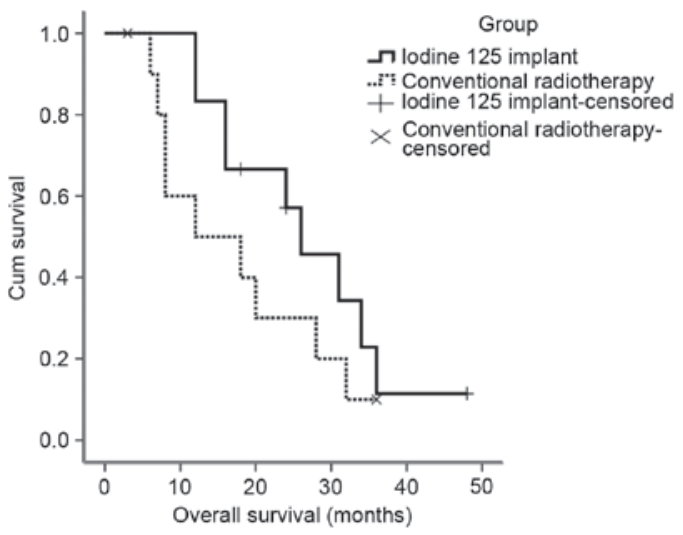

Figure 3. Comparison of the survival time in patients who underwent intraoperative ${ }^{125}$ I particle implantation and patients who underwent conventional radiotherapy. The survival rate of patients that underwent ${ }^{125} \mathrm{I}$ particle implantation was higher compared withthose that underwent postoperative external irradiation, particularly within 2 years of the operation. The overall survival rate did not differ significantly between the two groups.
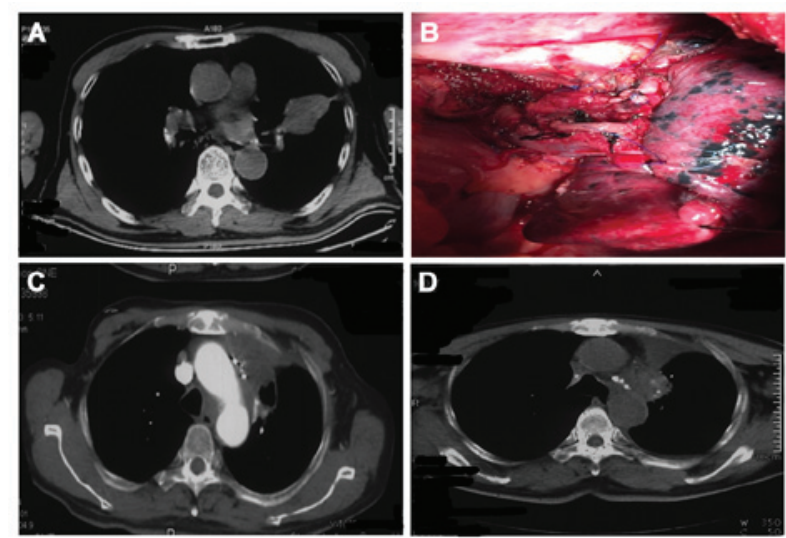

Figure 4. Treatment of a 72-year-old male patient (case 1). (A and B) CT Images prior to surgery. The patient underwent intraoperative ${ }^{125} \mathrm{I}$ particle implantation as macroscopic residual squamous carcinoma in the left upper lobe was found in the tissues beside the left main bronchus. Intraoperative ${ }^{125} \mathrm{I}$ particle implantation was performed at the bronchial stump and the peri-pulmonary artery areas. The left upper lobe was resected in October 2010, and $12^{125}$ particles $(29.6 \mathrm{MBq} /$ particle) were implanted at the site of cancer residual No metastasis was detected in the implantation regions during when the patient was re-examined 2 weeks' post-operation. The local control rate was satisfactory. (C and D) Images at 2 years following surgery. No recurrence in the implantation region was detected.

different between the two groups. In patients who underwent conventional treatment following R2resection, the median survival time was 12 months and the local control rate was $54.6 \%$ A meta-analysis of 9 studies revealed that in the majority of patients who underwent incomplete resection $(\mathrm{R} 1+\mathrm{R} 2)$, radiochemotherapy resulted in a median survival time of 6.5-19.1 months (7), which was consistent with the survival rate in the conventional treatment group in the current study.

The T-residual sizes during R2 resection are generally small. Therefore, intraoperative implantation with a small number of ${ }^{125} \mathrm{I}$ particles results in an effective dose of 100-120 Gy to the target region (1). ${ }^{125}$ I particle implantation provides sufficient radiation for the target regions, with minimal toxicity to the surrounding tissues $(1,8)$. Therefore, intraoperative implantation results in relatively high local control rates (4). In a previous study performed by Heelan et al (9) surgical treatment and intraoperative brachytherapy with $125 \mathrm{I}$ particles were used to treat patients with stage-IIIa NSCLC cancer with mediastinal lymph node metastases. The treatment resulted in an increase in the local control rate from 63 to $76 \%$. In a study performed by Lee et al (10), where 33 patients with lung cancer who were not candidates for lobectomy or pneumonectomy underwent limited resection, ${ }^{125} \mathrm{I}$ particles were implanted into the tissues forinternal irradiation. The findings of the study suggested that internal irradiation with ${ }^{125} \mathrm{I}$ particles reduces the recurrence rate in patients with lung cancer that are undergoing limited resection (10). A multicenter study revealed that, for selected NSCLC patients, sub-lobar resection combined with ${ }^{125}$ I particle implantation may result in similar local recurrence and survival rates, compared with lobectomy (11). Irradiation of the residual disease tissues with a low dose of ${ }^{125}$ I particles was effective, with a half-life of 4.5 years. For patients with low volume T-residuals ${ }^{125} \mathrm{I}$ particle implantation was comparable with $\mathrm{R} 0$ resection. In patients with simple T4 disease contraindicated for extended radical resection, ${ }^{125} \mathrm{I}$ particle implantation during $\mathrm{R} 2$ resection for local control results in improved outcomes compared with external irradiation. Control of the primary tumors by ${ }^{125}$ I particle implantation was able to reduce the risk of distant metastases and increase the survival rate $(12,13)$.

However, in patients with N2-residual disease, the metastatic lymph nodes were generally enlarged and fixed, and invasion of the pulmonary artery, superior vena cava and main bronchus was also present. In addition, these patients were generally diagnosed with advanced lung cancer, with the majority of patients succumbing to distant metastases and even extensive resection was associated with poor efficacy.

Therefore, effective control of the residual cancer made no significant difference to the survival rate and distant metastases when compared with patients without effective control. For patients with N2-residual disease, local control was effective. However, the extent of lymph node metastasis was generally wide. Unfortunately, the external radiation dose cannot generally exceed 60 Gy due to the tolerance limits of normal lung tissue. However, $60 \mathrm{~Gy}$ is a dose that is not sufficient for tumor eradication (14). The pulmonary volume of the patients was reduced following the operation, resulting in poor pulmonary function. The pulmonary V20 is generally $30 \%$ higher compared with the normal pulmonary volume, with a high risk of developing radiation pneumonitis (15).

Although increasing the dose of external irradiation is difficult due to the dose-tolerance limits of the normal lung tissue (4). ${ }^{125}$ I particle implantation is able to reduce the total radiation and increase the dosage in the irradiation region (1). When combined with external irradiation, ${ }^{125}$ I particle implantation may aid management of regions where N2 lymph node metastases are present. Future studies should therefore focus on methods, which can effectively control N-residual disease.

The safety profile of intraoperative ${ }^{125}$ I particle implantation, including the occurrence of bronchial stump fistula and major blood vessel rupture, and the radiation dose, are the most pressing surgical concerns. The pathological changes induced by NSCLC include shrinkage necrosis, while liquefactive necrosis and perforation are very rare (16). Other studies 
have reported small areas of fibrosis in the tissues around the ${ }^{125}$ I particles. However, the effects on pulmonary function were relatively low, and the safety profiles were higher for intraoperative ${ }^{125}$ I particle implantation compared with external radiation $(17,18)$. Therefore, ${ }^{125} \mathrm{I}$ particle implantation is a reliable treatment method for lung cancer. Trombetta et al (19) investigated 29 NSCLC patients with tumors adjacent to the aorta, and a ${ }^{125} \mathrm{I}$ particle mesh was used to cover the surface of the aorta for the treatment, suggesting that the treatment was safe and effective (6). The effective tumor irradiation time was four half-value period (59 days). An average of 10 particles were implanted during the operation. The radiation dose for the surgeons was $<200 \mu \mathrm{Sv}$ each time. This dose was demonstrated to be safe (20), and the cost for intraoperative ${ }^{125} \mathrm{I}$ particle implantation and postoperative radiotherapy was $\$ 1,000$ and $\$ 6,000$, respectively, in China. Additionally, for postoperative radiotherapy, one more month hospital stay is required, which is a marked improvement on postoperative radiotherapy.

In the present retrospective study, the results revealed a higher overall survival rate in the ${ }^{125} \mathrm{I}$ particle treatment group compared with the conventional treatment group within 2 years following surgery, while the difference after 2 years was not evident between the two treatment groups. The incidence of local control in the ${ }^{125} \mathrm{I}$ particle treatment group was significantly higher compared with the conventional group $(\mathrm{P}<0.05)$. Particularly for patients with simple T-residual disease, the intraoperative implantation of ${ }^{125} \mathrm{I}$ particles was able to significantly $(\mathrm{P}<0.05)$ reduce the tumor recurrence and increase the survival rate compared with conventional postoperative radiotherapy. Therefore, intraoperative ${ }^{125} \mathrm{I}$ particle implantation is a promising treatment option for NSCLC patients contraindicated for extended radical treatment.

The limitations of the current study relate to the relatively small number of patients and short follow-up time. A multi-center clinical trial with a larger sample size is required to confirm these data prior to routine clinical application. The findings of the present study revealed that, in lung cancer patients undergoing $\mathrm{R} 2$ resection, ${ }^{125} \mathrm{I}$ particle implantation at the regions of residual disease was able to improve patient outcomes. However, for patients with N-residual disease, the survival rate was not significantly different from those that have undergone conventional treatment.

\section{Acknowledgements}

The present study was supported by grants from the Sichuan Science and Technology Agency Science-Technology Support Project (grant no. ZCD162). The authors would like to thank Professor Shude Chai (Department of Thoracic Surgery, Tianjin Medical University, Tianjin, China) for technical assistance.

\section{References}

1. Li W, Dan G, Jiang J, Zheng Y, Zheng X and Deng D: Repeated iodine-125 seed implantations combined with external beam radiotherapy for the treatment of locally recurrent or metastatic stage III/IV non-small cell lung cancer: A retrospective study. Radiat Oncol 11: 119, 2016.
2. LoCicero JI: General Thoracic Surgery. Shields T, LoCicero JI, Reed C and Feins R (eds). 7th edition. Lippincott Williams \& Wilkins, Philadelphia, PA, pp1387-1425, 2009.

3. Foucault C, Mordant P, Grand B, Achour K, Arame A, Dujon A, Le Pimpec Barthes F and Riquet M: Unexpected extensions of non-small-cell lung cancer diagnosed during surgery: Revisiting exploratory thoracotomies and incomplete resections. Interact Cardiovasc Thorac Surg 16: 667-672, 2013.

4. Li W, Guan J, Yang L, Zheng X, Yu Y and Jiang J: Iodine-125 brachytherapy improved overall survival of patients with inoperable stage III/IV non-small cell lung cancer versus the conventional radiotherapy. Med Oncol 32: 395, 2015.

5. Tsim S, O'Dowd CA, Milroy R and Davidson S: Staging of non-small cell lung cancer (NSCLC): a review. Respir Med 104: $1767-1774,2010$.

6. Johnson M, Colonias A, Parda D, Trombetta M, Gayou O, Reitz B and Miften M: Dosimetric and technical aspects of intraoperative I-125 brachytherapy for stage I non-small cell lung cancer. Phys Med Biol 52: 1237-1245, 2007.

7. Dall K, Ford C, Fisher R and Dunning J: Is there a survival advantage of incomplete resection of non-small-cell lung cancer that is found to be unresectable at thoracotomy? Interact Cardiovasc Thorac Surg 16: 529-532, 2013.

8. Aye RW, Mate TP, Anderson HN, Johnson LP and Hill L: Extending the limits of lung cancer resection. Am J Surg 165: 572-576, 1993

9. Heelan RT, Hilaris BS, Anderson LL, Nori D, Martini N, Watson RC, Caravelli JF and Linares LA: Lung tumors: Percutaneousimplantation of I-125 sources with CT treatment planning. Radiology 164: 735-740, 1987.

10. Lee W, Daly BD, DiPetrillo TA, Morelli DM, Neuschatz AC, Morr J and Rivard MJ: Limited resection for non-small cell lung cancer: Observed local control with implantation of I-125 brachytherapy seeds. Ann Thorac Surg 75: 237-243, 2003.

11. Birdas TJ, Koehler RP, Colonias A, Trombetta M, Maley RH Jr, Landreneau RJ and Keenan RJ: Sublobar resection with brachytherapy versus lobectomy for stage Ib nonsmall cell lung cancer. Ann Thorac Surg 81: 434-439, 2006.

12. Cahlon O, Zelefsky MJ, Shippy A, Chan H, Fuks Z, Yamada Y, Hunt M, Greenstein S and Amols H: Ultra-high dose (86.4 Gy) IMRT for localized prostate cancer: toxicity and biochemicaloutcomes.IntJRadiatOncolBiolPhys 71:330-337,2008.

13. Zelefsky MJ, Yamada Y, Fuks Z, Zhang Z, Hunt M, Cahlon O, Park J and Shippy A: Long-term results of conformal radiotherapy for prostate cancer: Impact of dose escalation on biochemical tumor control and distant metastases-free survival outcomes. Int J Radiat Oncol Biol Phys 71: 1028-1033, 2008.

14. Langer M, Kijewski P, Brown R and Ha C: The effect on minimum tumor dose of restricting target-dose inhomogeneity in optimized three-dimensional treatment of lung cancer. Radiother Oncol 21: 245-256, 1991.

15. Kim TH, Cho KH, Pyo HR, Lee JS, Zo JI, Lee DH, Lee JM, Kim HY, Hwangbo B, Park SY, et al: Dose-volumetric parameters for predicting severe radiation pneumo-nitis after three-dimensional conformal radiation therapy for lung cancer. Radiology 235: 208-215, 2005.

16. Wang J, Wang J, Liao A, Zhuang $\mathrm{H}$ and Zhao Y: The direct biologic effects of radioactive $125 \mathrm{I}$ seeds on pancreatic cancer cells PANC-1, at continuous low-dose rates. Cancer Biother Radiopharm 24: 409-416, 2009.

17. Johnson M, Colonias A, Parda D, Trombetta M, Gayou O, Reitz B and Miften M: Dosimetric and technical aspects of intraoperative I-125 brachytherapy for stage I non-small cell lung cancer. Phys Med Biol 52: 1237-1245, 2007.

18. Chen H, Bao Y, Yu L, Jia R, Cheng W and Shao C: Comparison of cellular damage response to low-dose-rate 125I seed irradiation and high-dose-rate gamma irradiation in human lung cancer cells. Brachytherapy 11: 149-156, 2012.

19. Trombetta MG, Colonias A, Makishi D, Keenan R, Werts ED, Landreneau R and Parda DS: Tolerance of the aorta using intraoperative iodine-125 interstitial brachytherapy in cancer of the lung. Brachytherapy 7: 50-54, 2008.

20. Anglesio S, Calamia E, Fiandra C, Giglioli FR, Ragona R, Ricardi U and Ropolo R: Prostate brachytherapy with iodine-125 seeds: Radiation protection issues. Tumori 91: 335-338, 2005. 